\title{
Optimization Model of Searching Stratage in Sea
}

\author{
Chiyuan Ren ${ }^{1}$, Xin Tian ${ }^{2, *}$, Gang Zhu ${ }^{1}$ and Song Zou ${ }^{1}$ \\ ${ }^{1}$ Science College, Southwest Petroleum University, Chengdu, China \\ ${ }^{2}$ Applied technology College, Southwest Petroleum University, Chengdu, China \\ Corresponding author
}

\begin{abstract}
Searching a lost plane on seas usually is a task hard to implement in most situation, due to scarce data and widely sea. To overcome it, an optimization model is proposed. The proposed approach is based on the details and can govern the key information in the searching. Meanwhile it is easy to be understood and to be expanded. A plenty of numerical simulation is conducted to verify the approach. Numerical results show: the proposed approach can tract the flight and allocate the searching capability effectively.
\end{abstract}

Keywords-component; optimization; style; search strage; sea

\section{INTRODUCTION}

Air planes have become one of the most common transportation in our daily life. Recently, global commercial trades and international trips are more and more common and convenient as the economy develops [1-3]. Therefore, the risk of aviation accident has been inevitably raised. As a result, the importance of maritime search and rescue (SAR) is obvious in the way that it puts the last-ditch effort to prevent human life and property from suffering [4-6].

Aviation accident can be categorized into two types, which are communication emergency accident and accident whereby the contact is lost between the aircraft and air traffic controllers [7]. It is much easier locate the wreckage for the first type of accident as the aircraft still has its navigation and communication systems in operation. Nevertheless, once the aircraft losses contact with its ground controllers, it will become very difficult to find the jet which impedes the search process and further adds to the uncertainty for rescue. Unexpected huge loss of life and property may be hereby caused if the aircraft cannot be located fast and accurately [8].

Following by the research on the topic [9-11], a whole model is built. In this investigation, several concepts, including search capability, identification capability, search strength, possibility transition function, are induced, and then an optimization model is proposed to decide how to distribute the search ability of planes. It is worth noting that the possibility of a zone dynamically updated after each search, so that scheduling plan can be suitably adjusted under the circumstances.

\section{MODEL}

As described in the section above, we have estimated the possible wreckage zone. So the next task is to find out the true wreckage zone in the wreckage zone. In order to search the wreckage of place, usually some searching planes must be employed to perform searching above the sea. Because the number of searching plane is finite, it makes it is impossible to search whole possible wreckage with great searching strength. Usually, putting more searching in the zone with more possibility is advisable. An approach how to design the searching plan is proposed, and it can arrange the searching optimally. To illustrate our model, the relative processes must be explained clear firstly.

\section{A. Searching Capability}

Searching capability of a searching plane is the capability of the plane can find out whether a zone is wreckage zone or not in a flight. Usually, searching capability relates with the cruising time, cruising distance and searching equipments set up on the plane. The greater the cruising time and the cruising distance are, the greater the searching capability is. The more advanced the employed equipment is, the greater the searching capability. So based on some approximation, a simple model is provided to formulate this relation as:

$$
Q_{i}=S_{i} \cdot h_{i},
$$

where $Q_{i}, S_{i}, h_{i}$ are the searching capability, normal searching area, and searching time for a plane in a flight, respectively. The searching capabilities of the planes are listed as following.

TABLE I. THE SEARCH CAPABILITY OF THREE TYPES OF PLANES

\begin{tabular}{|c|c|c|c|}
\hline Airplane type & $\begin{array}{c}\text { Cruise time } \\
(h)\end{array}$ & $\begin{array}{c}\text { Normal search } \\
\text { area }\left(\mathrm{n} \cdot \mathrm{mile}^{2} / \mathrm{h}\right)\end{array}$ & $\begin{array}{c}\text { Search capability } \\
\left(n \text { mile }{ }^{2}\right)\end{array}$ \\
\hline$E-2 C I I$ & 4.26 & 180 & 766.8 \\
\hline$R A H-66$ & 5.25 & 220 & 1155 \\
\hline$U-2$ & 3.44 & 150 & 516 \\
\hline
\end{tabular}

B. Searching Strength

Planes are arranged to perform a searching in a zone. There are many choices can be employed. We can order a plan to search a very widely zone, or we can also order it to search a very small zone in one flight. Or we can order two planes to search a same zone at the same time. It implies a given zone can be searched with different strengths. It is called as searching strength in this investigation. In practice, the searching strength is the searching capability inputted to a unit zone in a flight, which can be represented as

$$
r_{i}=\frac{Q_{i}}{S_{i}},
$$


where $r_{i}, Q_{i}, S_{i}$ are the searching strength, searching capability inputted into, and area of zone $i$. Furthermore, the searching strength of a point $P$ can be also represented more mathematically. In order to implement it, we define the neighborhood of $P$ as $u(P, \delta)$, where $\delta$ is the radius of the neighborhood. Hence, the searching strength in the point $P$ can expressed as

$$
r(P)=\lim _{\delta \rightarrow 0} \frac{Q_{u(P, \delta)}}{S_{u(P, \delta)}},
$$

where $Q_{u(P, \delta)}, \mathrm{S}_{u(P, \delta)}$ are the searching capability inputted into, area in $u(P, \delta)$. Searching strength in a point is the key concept, based on it, an optimization model with continual variables is proposed in the investigation. It can make the proposed model more simply and strong.

\section{Identification Capability Function}

When we have performed a search in a zone with a searching strength, we can find out the zone is the true wreckage zone or not. But searching planes just probe a zone in the air, so usually their judgments are of uncertainty. Because the judgment maybe is wrong, so we employ a quantity to express the reliability of the judgment, which is named as identification capability. Generally speaking, the greater the searching strength is in a zone, the higher the identification capability of the search is in the zone. So the identification capability can be considered as the function of searching strength, i.e. $K=K(r)$. The function $K(r)$ can be set to different types, which is related to the many parameters in searching. Two simple but meaningful forms are proposed in this investigation.

\section{1) Marginal Effect Decreasing Type}

From the above analysis, we know: the greater the searching strength is in a zone, the higher the identification capability of the search is in the zone. But as the searching strength is increasing, the amplitude of identification capability increasing is not as great as the amplitude of searching strength. It usually is called as marginal effect decreasing principle in economics, which can be formulated in mathematics as

$$
\frac{d K}{d r}>0, \quad \frac{d^{2} K}{d r^{2}}<0
$$

An example is proposed in this investigation, which is $K(r)=\sqrt{r}$. And the result based on the function is simulated in the case study.

\section{2) Dirichlet type}

A more direct function is Dirichlet type function, which can be expressed as:

$$
K(r)=\left\{\begin{array}{ll}
\infty, & r \geq r_{0} \\
0, & \text { else }
\end{array},\right.
$$

where $r_{0}$ is a given value. The function indicates if the searching strength is greater than $r_{0}$ in a zone, then the judgment in the zone will be accepted, and vice versa. So it implies we should not input any searching capability with searching strength less than $r_{0}$.

Some more complex functions maybe be of stronger capacity to describe the between searching strength and identification capability. But more detail dataset will be needed, which is the further research content.

\section{Priori Possibility of the Possible Wreckage Zone}

Based on the locating model of possible wreckage zone, it is concluded that the possible wreckage zone is an approximate semicircle, which is named as $D$. The possible of a zone is the true wreckage zone is not even everywhere in $D$. Generally, the possible of the plane steers greater angle is less. So as depicted as Figure 1, we can set the priori possibility based the angle. The less the angle $\theta$ is, the greater the possibility of the zone is the true wreckage zone is.

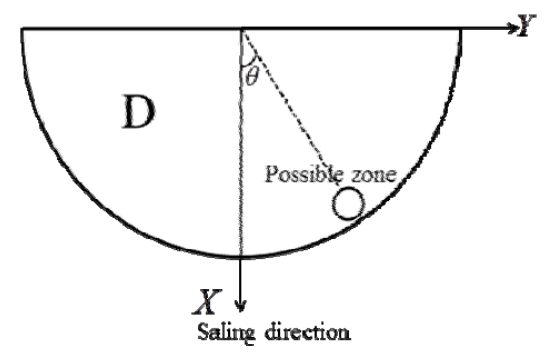

FIGURE I. $\quad \theta$ IS DEFINED AS AN ANGLE BETWEEN THE DIRECTION OF THE ORIGINAL FLYING LINE AND THE DIRECTION OF FLYING TO THE ZONE. THE SMALLER THE $\boldsymbol{\theta}$ IS, THE LARGER THE POSSIBILITY OF THE ZONE IS THE TRUE WRECKAGE ZONE IS.

An analyzed function is favor for the further investigation. A useful example is proposed, which is formulated as:

$$
\mathrm{p}_{0}(x, y)= \begin{cases}\frac{1}{2 \pi} e^{-\frac{\theta^{2}}{2}},(x, y) \in D \\ 0 \quad,(x, y) \notin D\end{cases}
$$

The priori possibility distribution function $p_{0}$ is depicted in Figure 2. It shows the zone along the fight direction on the last ping ring is of high possibility. 


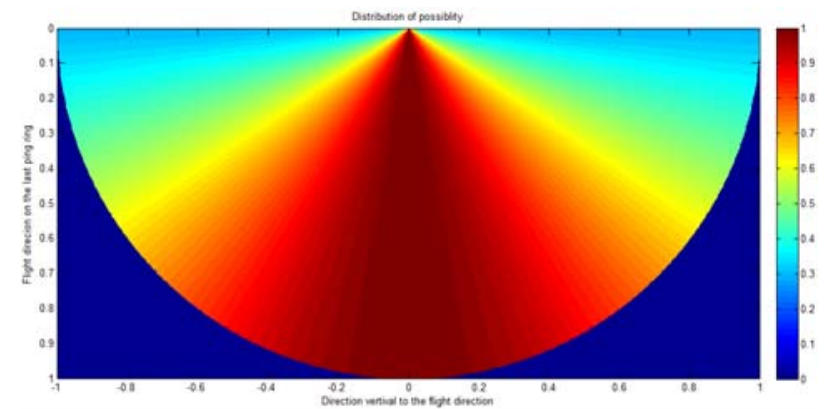

FIGURE II. DISTRIBUTION OF THE POSSIBILITY IN $D$. THE VERTICAL DIRECTION IS THE FLIGHT DIRECTION IN THE LAST PING RING. IF THE PATH OF THE FLIGHT IS CLOSER TO THE DIRECTION, THE POSSIBILITY IS GREATER.

\section{E. Possibility Transition Function}

According to the wreckage possibility distribution function, which describes distribution of the possibility, we will schedule our searching planes to find the true wreckage zone as soon as possible. Based on the priori possibility distribution function, an optimization searching plan will be designed. And then, the searching planes will perform the plan and return the searching results. After a search, a judgment will be made which indicates the zone is the true wreckage zone with greater possibility or less.

After completing a round of search, if the result shows the wreckage has been found, then the search is successful and the whole searching should be terminated. But unluckily, planes usually bring information back not so certainly. The positive information shows the zone is of higher possible, and the negative information indicates the opposite. So after a round of search, a more popular process is to update the possibility of the zone is the true wreckage zone. And how to update the prior possibility into posterior possibility is the content in this section. The updating principle includes two aspects.

1) If positive information shows the zone is the true wreckage zone, the posterior possibility should be greater than the priori one, and vice versa;

2) If the identification capability is greater, the adjusting extent is greater.

Many functions satisfy the two conditions above. A simple and feasible function is employed in this investigation, which can be formulated as:

$T(P)= \begin{cases}(1+\alpha)^{K}, & \text { point } \mathrm{P} \text { is judged as wreckage zone } \\ (1-\beta)^{K}, & \text { point } \mathrm{P} \text { is not judged as wreckage zone }\end{cases}$

where $\alpha$ and $\beta$ are positive real number, which are used to adjust the speed of updating, and $K$ is the identification capability, which changes the speed of updating distinctively.

\section{F. Posterior Possibility}

After a round of search, based on the searching results and the possibility transition function, the priori possibility is updated to posterior possibility. And the relation among them can be expressed as:

$$
p_{(i)}=p_{(i-1)} \cdot T,
$$

where $p_{i}$ is posterior possibility, and $p_{i-1}$ is priori possibility in the $i^{\text {th }}$ round of search. It implies our strategy can be conformed iteratively, and the possibility distribution function will be updated as iterations.

\section{OPTIMIZATION OF SEARCHING}

Based on the processes above, we describe the whole searching process and it can be implemented by five steps.

Step 1: Estimate the priori possibility in the possible wreckage zone.

Step 2: In this investigation, we do not make a plan for every plan, but we will make a plan about whole searching capability. It indicates we plan to solve how to allocate the searching capability in the possible wreckage zone, but not allocate the searching zone for every plane.

The searching plan is designed based on the priori possibility estimated in step1. An optimization plan must be made to allocate the searching capability for different zones and form different searching strength in the zones. In this investigation, we set our object to maximize the whole identification capability in $D$ for finding out the wreckage of the lost plane in a round. The model can be formulated as

$$
\begin{aligned}
& \max _{r} \int_{D} K \cdot p \\
& \text { s.t. } \int_{D} r=Q
\end{aligned}
$$

where $K$ is the identification capability function, which is related to search strength, $p$ is the priori possibility of wreckage at point $P$, and $Q$ is the whole searching capability of all planes. The model is to maximize the whole identification capability by adjusting the searching strength distribution function $r$. The only constraint is the limitation of total searching capability.

It is a function optimization problem, and can be solved by variational method. Under certain conditions, the optimization solution exists. We can utilize Lagrange function to solve the optimization model above, which is:

$$
L(r, \lambda)=\int_{D} K \cdot p+\lambda\left(\int_{D} r-Q\right)
$$

The stagnation point can be solved by the following equations.

$$
\left\{\begin{array}{l}
\frac{\partial L}{\partial r}=\int_{D}\left(\frac{d K}{d r} \cdot p+\lambda\right)=0 \\
\frac{\partial L}{\partial \lambda}=\int_{D} r-Q=0
\end{array}\right.
$$

The equations are different to be solved mathematically, when $K$ is the abstract function. We just show the results, when the identification capability is set to marginal effect decreasing type and Dirichlet type. 
1) $K$ is set to marginal effect decreasing type, i.e. $K=\sqrt{r}$. The solution of the optimization model can be solved as

$$
r=\frac{p^{2} Q}{\int_{D} p^{2}},
$$

which shows the searching capability should be allocated based on square of priori possibility.

2) $K$ is set to Dirichlet type. The optimization solution is

$$
r(P)= \begin{cases}r_{0}, & P \in D^{*} \\ 0, & P \notin D^{*}\end{cases}
$$

where $D^{*}$ satisfies

$$
\begin{aligned}
& \max \int_{D^{*}} p \\
& \text { s.t. } \\
& \int_{D^{*}} r_{0}=Q
\end{aligned}
$$

This formulation is seen to be very complex. But its real mean is simple, which is the whole searching capacity will be inputted into maximum possibility zone with searching strength equal to $r_{0}$.

Step 3: Perform searching based the plan above, and evaluate the searching result. Meanwhile, determine whether the search is over. If the wreckage of the lost plane is found or the searching number is up to the maximum, the all searching finishes.

Step 4: Compute possibility transition function based on the searching result and searching strength.

Step 5: Compute posterior possibility based on priori possibility and the possibility transition function. Then set the posterior possibility as the priori possibility, just to step 2 and perform the next round search.

The flowchart of the whole process is depicted as Figure 3.

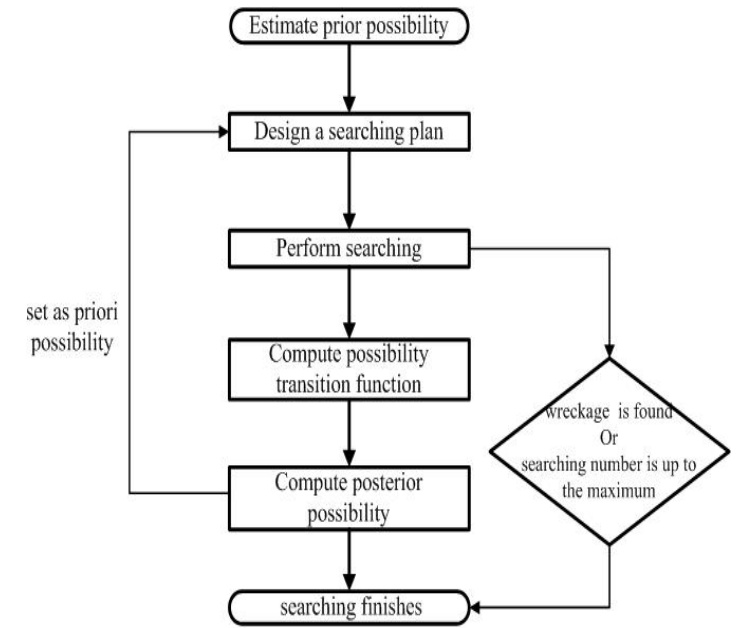

FIGURE III.

VISUAL DEPICTION OF SEARCHING STEP

\section{DISCUSSION \& CONCLUSION}

In this investigation, we have developed an optimal strategy to search the lost plane in the sea. The proposed approach is based on the details and can govern the key information in the searching. Meanwhile it is easy to be understood and to be expanded. The proposed optimization model is fit for the plenty of searching plane need to be planed. Furthermore, the more the searching planes is, the more distinct the advantage of the model is. Finally, a plenty of numerical simulation is conducted to verify the approach. Numerical results show: the proposed approach can tract the flight and allocate the searching capability effectively. And the wreckage can be found out more easily if the proposed approach is employed. The mainly conclusion can be listed as:

1) Some basic concepts, such as searching capacity, searching strength, identification capability function, priori possibility and so on, are defined. Based on them, we have proposed a continue optimization model to describe the allocation of searching capability.

2) We have solved the optimization model based on variation method in some condition. The analysis shows we should allocate the searching capability based on the square of possibility, when the marginal effect decreasing type identification function is employed. The simple conclusion is useful in practice.

\section{ACKNOWLEDGMENT}

The research is funded by Southwest Petroleum University.

\section{REFERENCES}

[1] Chen Ming Dong.(2007).Research on Maritime Searching Techniques.

[2] Duncan Steel.( 2014).Potential Terminal Locations for MH370. http://www.duncansteel.com/archives/666

[3] Duncan Steel.(2014). Further Notes on Ping Rings and the Search for MH370. http://www.duncansteel.com/archives/710

[4] Chris Ashton, Alan Shuster Bruce, Gary Colledge, Mark Dickinson.The Search for MH370.Journal of Navigation, 2014, Vol.68 (1), pp.1-22

[5] Xing Shengwei.(2012).Research on Global Optimization Model and Simulation of Joint Aeronautical and Martime Search.

[6] Koopman BO. The theory of search. The optimum distribution of searching effort. Management Science,1957,5(5):613-626.

[7] Charnes A, Cooper W W. The theory of search: optimum distribution of search effort. Management Science, 1958, 5(1):44-50.

[8] De Guenin J. Optimum distribution of effort: An extension of the Koopman basic theory. Operations Research, 1961, 9(1): 1-7.

[9] Stone L D. Search for targets with generalized conditionally deterministic motion, SIAM Journal on Applied Mathematics, 1977, 33(3): 239-255.

[10] Cooper D C, Frost J R, Robe R Q. Compatibility of land SAR Procedures with search theory. Washington D. C.: U.S. Coast Guard,2003.

[11] Trummel K E, Weisinger J R. The complexity of the optimal searcher path problem. Operations Research, 1986,34(2):324-327. 